\title{
Deterioration of left ventricular function following atrio-ventricular node ablation and right ventricular apical pacing in patients with permanent atrial fibrillation
}

\author{
T. Szili-Torok, G. P. Kimman, D. Theuns, D. Poldermans, J. R. T. C. Roelandt and \\ L. J. Jordaens
}

Department of Cardiology, Thoraxcentre, Erasmus Medical Centre Rotterdam, Rotterdam, The Netherlands

\begin{abstract}
Aims Transcatheter radiofrequency ablation of the atrioventricular (AV) node followed by ventricular pacing has been shown to improve symptoms and quality of life of patients with atrial fibrillation (AF). It is assumed that function improves, but this has been less well demonstrated. The aim of this study was to assess the long-term effect of AV node ablation and ventricular pacing on left ventricular ejection fraction (LVEF) in patients with permanent AF.
\end{abstract}

Methods and Results All 12 patients studied had permanent AF for at least 12 months (mean age 70 years, range 41 to 78). LVEF was determined 6 days and 3 months after AV node ablation by radionuclide ventriculography, at a paced rate of 80 beats $\min ^{-1}$. Cardiac dimensions were measured by means of transthoracic echocardiography. No major changes in pharmacological therapy were made during 3 months follow-up period. LVEF showed a significant deterioration after 3 months follow-up period for the group $(47 \cdot 5 \pm 14 \cdot 4 \% ; 6$ days after ablation vs $43 \cdot 2 \pm 13 \cdot 7 \%$; 3 months after ablation, $P<0 \cdot 05)$. There were no significant differences in left ventricular cavity dimensions directly after AV node ablation and 3 months later (LVEDD $51 \cdot 2 \pm 10 \cdot 7 \mathrm{~mm}$ vs $52.6 \pm 8.6 \mathrm{~mm}, P=\mathrm{NS}$; LVESD: $36 \cdot 1 \pm$ $14.2 \mathrm{~mm}$ vs $36 \cdot 6 \pm 9 \cdot 7 \mathrm{~mm}, P=\mathrm{NS})$. Left atrial size did not show reduction 3 months after AV node ablation $(50 \cdot 8 \pm 13 \cdot 6 \mathrm{~mm}$ vs $51 \cdot 0 \pm 14 \cdot 1 \mathrm{~mm}, P=\mathrm{NS})$.

Conclusion The restoration of a regular ventricular rhythm following AV node ablation for patients in permanent AF does not result in improvement in left ventricular function.

(Europace 2002; 4: 61-65)

(C) 2002 The European Society of Cardiology

Key Words: Atrial fibrillation, ablation, atrioventricular node, cardiac function.

\section{Introduction}

Atrial fibrillation (AF) is a common supraventricular arrhythmia, which leads to cardiac dilatation and dysfunction $^{[1]}$. Theoretically, the management of this arrhythmia including restoration of sinus rhythm or regular ventricular paced rhythm should result in an improvement in the patient's symptoms as well as in cardiac function. Some authors advocate achieving this by ablating the atrio-ventricular (AV) node followed

Manuscript submitted 21 January 2001, accepted after revision 20 October 2001.

Correspondence: Tamas Szili-Torok, MD, Department of Clinical Electrophysiology, Thoraxcentre, Rotterdam, Dr Molewaterplein 40, 3015 GM, Rotterdam, The Netherlands. E-mail: szili@card.azr.nl by right ventricular (RV) apical pacing. Although the advantage of a regular ventricular response seems to be important ${ }^{[2]}, \mathrm{RV}$ apical stimulation is not physiological because normal ventricular activation along the natural conduction system is bypassed and the ventricles are activated in an abnormal sequence ${ }^{[3,4]}$. Chronic apical ventricular pacing is associated with myocardial cellular changes and leads to dysfunction of the left ventricle ${ }^{[5,6]}$. However, some studies, including a prospective multicentre randomized trial, indicated that exercise tolerance and the quality of life improved after AV node ablation and RV apical pacing ${ }^{[7-9]}$. The reversibility of cardiac dysfunction after such intervention was one of the reasons that the concept of tachycardiomyopathy was introduced. However, it is clear that when atrial function is lost, ventricular performance will suffer in the long term. Since only limited and confusing data are available 
Table 1 Clinical and demographic characteristics of the patients

\begin{tabular}{rrrrccc}
\hline Pt. no. & Age & Sex & $\begin{array}{c}\text { Duration of AF } \\
\text { (months) }\end{array}$ & $\begin{array}{c}\text { QRS duration } \\
\text { (s) }\end{array}$ & Cardiac disease & Medication \\
\hline 1 & 67 & F & 12 & $0 \cdot 12$ & SSS & $3,4,7,8$ \\
2 & 68 & M & 108 & $0 \cdot 08$ & DCMP & $2,3,4,6$ \\
3 & 66 & M & 38 & $0 \cdot 14$ & Atrial flutter & $4,6,7$ \\
4 & 78 & F & 12 & $0 \cdot 12$ & Atrial flutter & $2,3,4,6$ \\
5 & 70 & F & 24 & $0 \cdot 08$ & Hypertension & $4,7,8$ \\
6 & 74 & F & 48 & $0 \cdot 12$ & ASD & $1,3,8$ \\
7 & 66 & M & 48 & $0 \cdot 08$ & Non obstr. HCMP & $1,2,3,6$ \\
8 & 41 & M & 48 & $0 \cdot 08$ & VHD & $2,4,7$ \\
9 & 67 & F & 12 & $0 \cdot 12$ & SSS, Atrial flutter, Hypertension & $1,4,7,8$ \\
10 & 55 & M & 156 & $0 \cdot 08$ & VHD & $2,3,4$ \\
11 & 63 & M & 12 & $0 \cdot 16$ & Hypertension & $2,3,4,7,8$ \\
12 & 76 & F & 16 & $0 \cdot 12$ & - & 4,8 \\
& & & & & &
\end{tabular}

$\mathrm{SSS}=$ sick sinus syndrome; $\mathrm{AF}=$ atrial fibrillation; $\mathrm{F}=$ female; $\mathrm{M}=$ male; $\mathrm{DCMP}=$ dilatative cardiomyopathy; $\mathrm{ASD}=$ atrial septal defect; $\mathrm{HCMP}=$ hypertrophic cardiomyopathy; $\mathrm{VHD}=$ valvular heart disease; in medication: $1=$ beta blocker; $2=\mathrm{ACE}$ inhibitor; $3=$ diuretics; $4=$ digoxin; $5=$ vasodilators; $6=$ amiodarone; $7=\mathrm{Ca}$ channel blocker; $8=$ other antiarrhythmics.

on the long term effect of AV node ablation and RV apical pacing, we aimed to conduct a study to assess long term effects of AV node ablation and RV apical pacing on the left ventricular performance using a completely different approach from previous investigators. To exclude the possible bias ${ }^{[2,10,11]}$ of comparing pre- and post-ablation states respectively in fast conducted AF and paced rhythm, we determined the left ventricular ejection fraction (LVEF) 6 days after (not before) and 3 months after AV node ablation in 12 patients with permanent AF.

\section{Methods}

Patients were eligible if they had permanent AF and if the ventricular rate could not be adequately controlled by drug therapy. From February 1999 to February 2000, 12 patients with permanent $\mathrm{AF}$ underwent ablation of the AV node and insertion of a VVIR pacemaker and $\mathrm{RV}$ apical pacing. There were six men and six women with an age range from 41 to 78 (mean 70) at the time of ablation.

\section{Study patients}

The clinical and demographic characteristics of the patients are shown in Table 1. All patients were in NYHA functional class II or III. The type of AF was permanent in all 12 patients. Only two patients had an increased QRS duration (140-160 ms) before AV node ablation. All had complete heart block after the ablation, with QRS duration less than $120 \mathrm{~ms}$ in $8 / 12$ patients. There were no major changes in medication during the 3 month follow up period. VVIR pacing with rate responsive mode was used in all patients. For
LVEF measurements VVI $80 \mathrm{bpm}$ pacing mode was temporarily programmed $1 \mathrm{~h}$ before measurement.

\section{Ablation procedure}

A temporary pacing electrode was inserted via a femoral vein into the RV before the ablation procedure. One patient already had a permanent pacemaker inserted. Third degree AV block was achieved using a conventional right sided approach in every case with radiofrequency energy. Webster D or F curve thermocouple mapping/ablation catheters were used to map the antero- and/or mid-septal region and to deliver radiofrequency (RF) energy. A permanent cardiac pacemaker was inserted $30 \mathrm{~min}$ after successful ablation. No major or minor complications related to RF ablation and pacemaker insertion were observed. Patients were subsequently followed-up in the outpatient clinic.

\section{Evaluation of left ventricular ejection fraction ( LVEF)}

LVEF was measured with radionuclide ventriculography (red blood cells, marked with Technetium ${ }^{99}$ pertechnetate, $25 \mathrm{mCi}$ ). Imaging was performed in $45^{\circ}$ left anterior oblique (LAO). The $\mathrm{R}$ wave was used for gating, and 16-24 frames per cycle were stored until 400000 counts per image were acquired. Measurement was made 6 days after and 3 months after the ablation procedure, always at the paced rate of $80 \mathrm{bpm}$.

\section{Echocardiographic measurements}

M-mode and cross-sectional echocardiograms were obtained at the time of measurement of LVEF by 


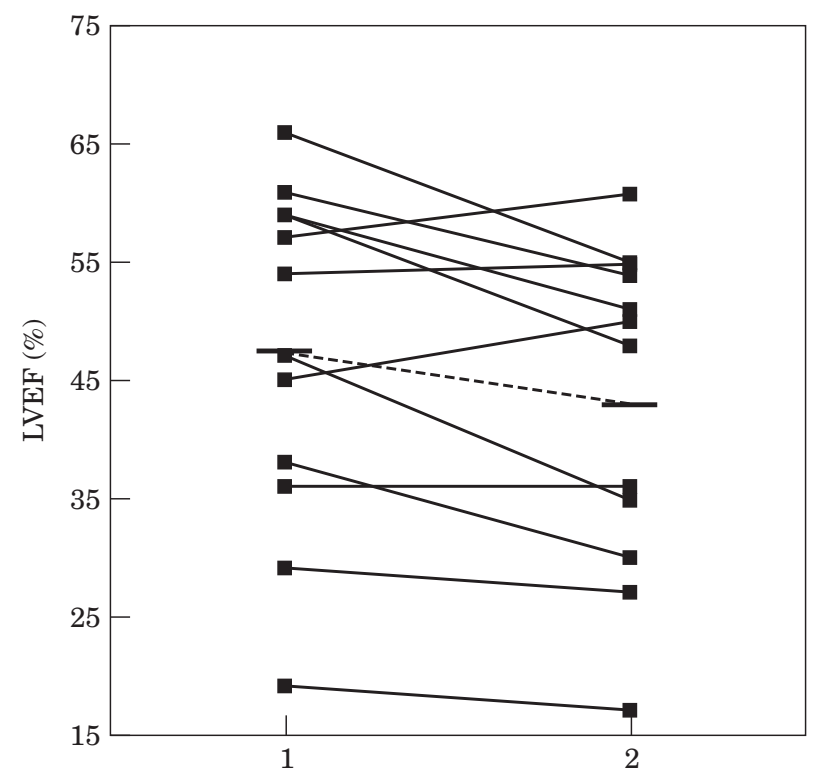

Figure 1 Individual representation of left ventricular ejection fraction (LVEF) 6 days after (1) and 3 months (2) after AV node ablation.

radionuclide ventriculography. Left atrial (LA) size, end systolic (LVESD) and end diastolic diameters (LVEDD) were measured according to the recommendations of the American Society of Echocardiography. Fractional shortening was calculated according to the following formulae: $\mathrm{FS} \%=($ LVEDD-LVESD $) /$ LVEDD $\times 100$. In all patients the echocardiograms were recorded in the same paced $(80 \mathrm{bpm})$ rhythm. After analysis the pacemaker was reprogrammed to VVIR mode.

Statistical analysis: The measured values are expressed as mean $\pm \mathrm{SD}$. Non-parametric data were compared using the Wilcoxon test. Correlation analysis was performed using the Pearson test. The level of significance was set at $0 \cdot 05$.

\section{Results}

\section{Effect of AF on left ventricular function}

The initial LVEF was lower in patients with longer duration of $\mathrm{AF}(\mathrm{r}=-0.74, P<0.05)$. A longer duration of AF was associated with larger LVEDD dimension $(\mathrm{r}=-0.833, \quad P<0.01)$ and LVESD dimension $(\mathrm{r}=$ $-0 \cdot 795, P<0 \cdot 05)$.

\section{Effect of pace and ablate therapy on left ventricular function}

The mean LVEF decreased from $47 \cdot 5 \pm 14 \cdot 4 \%$ to $43 \cdot 2 \pm 13 \cdot 7 \% \quad(P<0 \cdot 05)$. Only two patients showed obvious $(>5 \%)$ improvement in LVEF (Fig. 1). Left ventricular dimensions did not show significant change during 3 months follow up (Table 2).

\section{Left atrial dimensions}

LA dimensions did not show changes after AV node ablation and RV apical pacing (Table 2).

\section{Fractional shortening (FS)}

Fractional shortening did not show significant changes 6 days and 3 months after AV node ablation.

\section{Discussion}

The major finding of our study was that LVEF deteriorated 3 months after AV node ablation with chronic RV apical pacing. Secondly, the LV dimensions and the LA size did not show significant changes during the study period. Our data confirm the hypothesis that LV function becomes depressed after long term duration of AF.

\section{Causes of $L V$ dysfunction in patients with atrial fibrillation}

It is now generally accepted that supraventricular incessant arrhythmias can lead to myocardial dysfunction and dilation ${ }^{[1]}$. Factors contributing to HF when atrial arrhythmias are present are the loss of an effective, well timed atrial contraction and the fast and irregular ventricular response. These factors impair diastolic function and finally may lead to tachycardiomyopathy ${ }^{[1]}$. Inappropriately fast heart rates occur even during minor exercise in patients with atrial fibrillation. This is accompanied by depressed heart rate at peak exercise in cases of left ventricular dysfunction ${ }^{[12-14]}$. Therefore, the aims of improving function of the patients can be reached by restoration of sinus rhythm, but also (to a less complete extent) by control of the ventricular rate. It is believed that rate control, with drugs or RF catheter ablation alone is sufficient to improve the outlook of these patients. However, evidence has been presented that rhythm control in heart failure has disadvantages ${ }^{[15]}$. Several investigators have considered it acceptable to randomize patients to rhythm or rate control, as many questions remain unanswered ${ }^{[16]}$.

\section{$L V$ function after $A V$ node ablation}

After ablation, pacemaker dependency is the rule, with implantation of a VVIR system when atrial electrical activity is no longer thought to be present. Although the advantage of a regular ventricular response is important ${ }^{[2]}, \mathrm{RV}$ apical stimulation is not physiological because normal ventricular activation along the natural 
Table 2 Echocardiographic findings at the time and 3 months after $R F$ ablation of AV node

\begin{tabular}{ccc}
$\begin{array}{c}\text { Six days after } \\
\text { ablation }(\mathrm{mm})\end{array}$ & $\begin{array}{c}\text { Three months after } \\
\text { ablation }(\mathrm{mm})\end{array}$ & $P$ value \\
$50 \cdot 8 \pm 13 \cdot 6$ & $51 \cdot 0 \pm 14 \cdot 1$ & $\mathrm{NS}$ \\
$51 \cdot 2 \pm 10 \cdot 7$ & $52 \cdot 6 \pm 8 \cdot 6$ & $\mathrm{NS}$ \\
$36 \cdot 1 \pm 14 \cdot 2$ & $36 \cdot 6 \pm 9 \cdot 7$ & $\mathrm{NS}$ \\
$31 \pm 0 \cdot 1$ & $31 \pm 0 \cdot 1$ & $\mathrm{NS}$ \\
\hline
\end{tabular}

$\mathrm{LA}=$ left atrial; $\mathrm{LV}=$ left ventricular; $\mathrm{NS}=$ non significant.

conduction system is not present or bypassed and the ventricles are activated in an abnormal sequence ${ }^{[3,4]}$. Some investigators found significant improvement in left ventricular function after AV node ablation and RV apical pacing. Heinz et al. reported improvement of LV function after AV node ablation in patients with chronic $\mathrm{AF}$ and atrial flutter. This was attributed to decrease in end-systolic dimension ${ }^{[17]}$. Rodriguez and co-workers showed a beneficial effect of the 'ablate and pace' therapy in patients with lone $\mathrm{AF}$ in the subgroup where the baseline LVEF was lower than $50 \%$. They also showed a significant decrease in LV end systolic and end diastolic dimensions after ablation ${ }^{[7]}$. A long term improvement in systolic and diastolic left ventricular function was reported by Edner et al. in patients with left ventricular dysfunction ${ }^{[18]}$. Ablation of the AV node had no adverse effect on normal left ventricular function. In contrast to these findings, in the present study the LVEF decreased independently from the initial value (Fig. 1). In a recent study by Redfield et al. 63 patients underwent AV node ablation and pacing therapy with atrial fibrillation and reduced systolic function. Sixteen patients $(25 \%)$ had improvement in systolic function after AV node ablation ${ }^{[19]}$. These results lead to the concept of tachycardia-related cardiomyopathy which diagnosis should be considered in all patients in whom systolic dysfunction occurs subsequent to atrial fibrillation. More convincing are some studies showing that quality of life indices significantly improved after ablation of the atrio-ventricular node ${ }^{[9]}$. In a prospective multicentre randomized study of Brignole and co-workers a mixed population of patients with $\mathrm{AF}$ and HF was recruited. The 'ablate and pace' therapy was superior to drug therapy in controlling symptoms, but the efficacy appeared to be less than was observed in previous uncontrolled studies. Importantly, objective cardiac performance did not show any improvement by the treatment. However, less hospital admissions and less physician visits were necessary after ablation ${ }^{[8]}$.

\section{Methodological problems}

The three major differences between the former and present studies are: the timing of the measurements, the composition of the study group and the method used for the measurement of LVEF. Some authors described that less than $40 \%$ of their patients had heart failure, measured with some more or less objective parameters ${ }^{[8]}$. Our study group was composed of patients with long standing AF, who all had signs of moderate to severe heart failure. Concerning the timing, all of the significant former studies compared LV function before and after AV node ablation ${ }^{[7-9,17,18]}$. Some studies were retrospective and the timing of the measurement of LVEF varied in a wide time range ${ }^{[7,17,18]}$. In our prospective study the timing was more appropriate. We tried to minimize the effects of possible acute haemodynamic changes caused by a restoration of a regular ventricular response. Therefore we compared the LVEF 6 days and 3 months after AV node ablation and pacemaker implantation exactly under the same conditions. One of the other possible explanations for the difference between the results of previous and the present study could be the significant difference in measurements. In this study, LVEF was measured by means of radionuclide ventriculography (RNV). RNV is highly reproducible and has an error of less than 3\%. Probably RNV is less sensitive to the errors caused by the change in ventricular geometry during RV pacing. The formula used for routine calculation of LVEF during transthoracic echocardiography is based on constant ventricular geometry during contraction. The shape of the ventricle during RV apical pacing is changing, therefore a planimetry based measurement is more appropriate for the evaluation of LV function. We emphasize the importance of using RNV in further pacing studies assessing left ventricular function. Furthermore, none of the former investigators evaluated left ventricular performance exactly under the same conditions immediately and 3 months after AV node ablation ${ }^{[7-9,17,18]}$. Both assessments of LVEF in this study were measured during the same 80 beats . min $^{-1}$ paced cardiac rhythm.

\section{Survival after $A V$ node ablation}

Finally, the natural history of patients treated by AV node ablation is still not known. Sudden death has been reported in some subgroups of patients. It was first attributed to the technique of DC shock ablation ${ }^{[20]}$. However, it was also observed after RF ablation ${ }^{[21,22]}$. In a meta-analysis of 21 studies with a total of 1181 patients by Wood et al. the ablation and pacing therapy 
showed that the calculated 1-year mortality was comparable with medical therapy ${ }^{[23]}$. A considerable proportion of these deaths can be related to the acute effects of AV node ablation with QT prolongation and slow pacing rate and/or unreliable escape rhythms ${ }^{[21,24]}$. Another subgroup of deaths was reported late after AV node ablation, mainly in patients with $\mathrm{HF}^{[21,25]}$. The deterioration of pump function late after $\mathrm{AV}$ node ablation may play a role in this mortality. Therefore, careful adjustment of the medical therapy for these patients with LV dysfunction is very important including beta-blocker therapy ACE inhibitors, adjustment of electrolytes and avoidance of antiarrhythmic therapy ${ }^{[26,27]}$. The follow up of these patients must not be limited to the technical control of pacemaker function.

\section{Limitations of study}

This study is of a relatively small number of patients. The change in ejection fraction is statistically significant, but may not have great clinical significance. A factor influencing this may have been the long duration of atrial fibrillation in most of the patients with consequent lack of ability to recover. Quality of life measures were not employed in this work. Future studies should include such measures to achieve more clinical relevance of the left ventricular function data. Follow-up of the patients in this report is only 3 months and the longterm effects on left ventricular function remain to be ascertained.

\section{References}

[1] Gajewski J, Singer RB. Mortality in an insured population with atrial fibrillation. J Am Med Assoc 1981; 254: 1540-4.

[2] Daoud EG, Weiss R, Bahu M, et al. Effect of irregular ventricular rhythm on cardiac output. Am J Cardiol 1996; 78: 1433-6.

[3] Tse HF, Lau CP. Long term effect of right ventricular pacing on myocardial perfusion and function. J Am Coll Cardiol 1997; 29: 744-9.

[4] Little WC, Reeves RC, Arciniegas J, Katholi RE, Rogers EW. Mechanism of abnormal interventricular septal motion during delayed left ventricular activation. Circulation 1982; 65: 148691.

[5] Adomian GE, Beazell J. Myofibrillar disarray produced in normal hearts by chronic electrical pacing. Am Heart J 1986; 112: 79-83.

[6] Karpawich PP, Rabah R, Haas JE. Altered cardiac histology following apical right ventricular pacing in patients with congenital atrioventricular block. Pacing Clin Electrophysiol 1999; 22: 1372-7.

[7] Rodriguez LM, Smeets JRLM, Xie B, et al. Improvement in left ventricular function by ablation of atrioventricular nodal conduction in selected patients with lone atrial fibrillation. Am J Cardiol 1993; 72: 1137-41.

[8] Brignole M, Menozzi C, Gianfranchi L, et al. Assessment of atrioventricular junction ablation and VVIR pacemaker versus pharmacological treatment in patients with heart failure and chronic atrial fibrillation. A randomised controlled study. Circulation 1998; 98: 953-60.

[9] Natale A, Zimerman L, Tomassoni G, et al. AV node ablation and pacemaker implantation after withdrawal of effective rate control medications for chronic atrial fibrillation: effect on quality of life and exercise performance. Pacing Clin Electrophysiol 1999; 22: 1634-9.

[10] Lau CP, Leung WH, Wong CK, Cheng CH. Haemodynamics of induced atrial fibrillation: A comparative assessment with sinus rhythm, atrial and ventricular pacing. Eur Heart J 1990; 11: $219-24$.

[11] Naito M, David D, Michelson EL, Schaffenburg M, Dreifus LS. The hemodynamic consequences of cardiac arrhythmias: evaluation of the relative roles of abnormal atrioventricular sequencing, irregularity of ventricular rhythm and atrial fibrillation in a canine model. Am Heart J 1983; 106: 284-91.

[12] van den Berg MP, Crijns HJ, Gosselink AT, et al. Chronotropic response to exercise in patients with atrial fibrillation: relation to functional state. Br Heart J 1993; 70: $150-3$.

[13] Resnekov L, McDonald L. Electroversion of lone atrial fibrillation and flutter including hemodynamic studies at rest and on exercise. Br Heart J 1971; 33: 339-50.

[14] Corbelli R, Masterson M, Wilkoff BL. Chronotropic response to exercise in patients with atrial fibrillation. Pacing Clin Electrophysiol 1990; 13: 179-87.

[15] Flaker GC, Blackshear JL, McBride R, Kronmal RA, Halperin JL, Hart RG. Antiarrhythmic drug therapy and cardiac mortality in atrial fibrillation. The Stroke Prevention in Atrial Fibrillation Investigators. J Am Coll Cardiol 1992; 20: $527-32$.

[16] Wyse DG. The AFFIRM trial: Main trial and substudies what can we expect? J Interv Card Electrophysiol 2000; 4(Suppl. 1): 171-6.

[17] Heinz G, Siosrzonek P, Kreiner G, Gossinger H. Improvement in left ventricular systolic function after successful radiofrequency His bundle ablation for drug refractory, chronic atrial fibrillation and recurrent atrial flutter. Am J Cardiol 1992; 69: 489-92.

[18] Edner M, Caidahl K, Bergfeldt L, Darpö B, Edvardsson N, Rosenqvist M. Prospective study of left ventricular function after radiofrequency ablation of atrioventricular junction in patients with atrial fibrillation. Br Heart J 1995; 74: 261-7.

[19] Redfield MM, Kay GN, Jenkins LS, Mianulli M, Jensen N, Ellenbogen KA. Tachycardia-related cardiomyopathy: a common cause of ventricular dysfunction in patients with atrial fibrillation referred for atriovantricular ablation. Mayo Clin Proc 2000; 75: 790-5.

[20] Evans GT, Scheinman MM and the Executive Committee of the Registry. The percutaneous cardiac mapping and ablation registry: final summary of results. Pacing Clin Electrophysiol 1988;11: 1621-6.

[21] Jordaens L, Rubbens L, Vertongen P. Sudden death and long term survival after ablation of the atrioventricular junction. Eur J Cardiac Pacing Electrophysiol 1993; 3: 232-7.

[22] Olgin JE, Scheinman MM. Comparison of high energy direct current and radiofrequency catheter ablation of the atrioventricular junction. J Am Coll Cardiol 1993; 21: 557-64.

[23] Wood MA, Brown-Mahoney C, Kay N, Ellenbogen KA. Clinical outcomes after ablation and pacing therapy for atrial fibrillation. Circulation 2000; 101: 1138-44.

[24] Geelen P, Brugada J, Andries E, Brugada P. Ventricular fibrillation and sudden death after radiofrequency catheter ablation of the atrioventricular junction. Pacing Clin Electrophysiol 1997; 20: 343-8.

[25] Darpö B, Walfridsson H, Aunes M, et al. Incidence of sudden death after radiofrequency ablation of the atrioventricular junction for atrial fibrillation. Am J Cardiol 1997; 80: 1174-7.

[26] Flather MD, Yusuf S, Kober L, et al. Long-term ACEinhibitor therapy in patients with heart failure or leftventricular dysfunction: a systematic overview of data from individual patients. ACE-Inhibitor Myocardial Infarction Collaborative Group. Lancet 2000; 355: 1575-81.

[27] Lechat P, Escolano S, Golmard JL, et al. Prognostic value of bisoprolol-induced hemodynamic effects in heart failure during the Cardiac Insufficiency Bisoprolol Study (CIBIS). Circulation 1997; 96: 2197-205. 